\title{
Critical roles of specimen type and temperature before and during fixation in the detection of phosphoproteins in breast cancer tissues
}

\author{
Sibylle Gündisch ${ }^{1,5}$, Laura Annaratone ${ }^{2,5}$, Christian Beese ${ }^{1}$, Enken Drecol', Caterina Marchiò ${ }^{2,3}$, Elena Quaglino ${ }^{4}$, \\ Anna Sapino ${ }^{2,3}$, Karl-Friedrich Becker ${ }^{1}$ and Gianni Bussolati ${ }^{2}$
}

The most efficient approach for therapy selection to inhibit the deregulated kinases in cancer tissues is to measure their phosphorylation status prior to the treatment. The aim of our study was to evaluate the influence of pre-analytical parameters (cold ischemia time, temperature before and during tissue fixation, and sample type) on the levels of proteins and phosphoproteins in breast cancer tissues, focusing on the PI3 kinase/AKT pathway. The BALB-neuT mouse breast cancer model expressing HER2 and PAKT proteins and human biopsy and resection specimens were analyzed. By using quantitative reverse phase protein arrays (RPPA), 9 proteins and 16 phosphoproteins relevant to breast cancer biology were assessed. Cold temperatures before and during fixation resulted in a marked improvement in the preservation of the reactivity of biological markers (eg, ER, HER2) in general and, specifically, pHER2 and pAKT. Some phosphoproteins, eg, pHER2 and pAKT, were more sensitive to prolonged cold ischemia times than others (eg, pS6RP and pSTAT5). By comparing the phosphoprotein levels in core needle biopsies with those in resection specimens, we found a marked decrease in many phosphoproteins in the latter. Cold conditions can improve the preservation of proteins and phosphoproteins in breast cancer tissues. Biopsies $\leq 1 \mathrm{~mm}$ in size are the preferred sample type for assessing the activity of deregulated kinases for personalized cancer treatments because the phosphoprotein levels are better preserved compared with resection specimens. Each potential new (phospho)protein biomarker should be tested for its sensitivity to pre-analytical processing prior to the development of a diagnostic assay.

Laboratory Investigation (2015) 95, 561-571; doi:10.1038/labinvest.2015.37; published online 2 March 2015

In the era of personalized therapies, a precise assessment of biomarkers, both at the protein and gene levels, is crucial for an optimal patient management. ${ }^{1}$ This is especially true for breast cancer, where treatment is determined according to the levels of immunohistochemical (IHC) expression of hormone receptors and $\mathrm{HER}^{2}$ evaluated in formalin-fixed and paraffin-embedded (FFPE) tissues. However, variations in tissue processing affect the preservation of antigenic sites, preventing a reproducible measurement of proteins and phosphorylated epitopes.

Studies on the accuracy of the analytical results have highlighted the impact of a range of variables related to the pre-analytical phase before the molecular test is performed. ${ }^{3-6}$ Although a large number of variables potentially affect the tissue quality, ${ }^{7}$ recent studies have shown that cold ischemic time (ie, time from tissue removal to formalin fixation $)^{8,9}$ and temperatures during fixation processes ${ }^{10,11}$ are crucial. ${ }^{12,13}$

These parameters are particularly critical (also in terms of clinical impact) for the analysis of HER2 levels ${ }^{3}$ and for maintaining the antigenicity of phosphorylated proteins. ${ }^{3,14,15}$

In our experience, the temperature of tissue fixation may affect the preservation of biomarkers as well. This concept is consistent with the definite advantages offered by the preservation of fresh (ie, not fixed) surgical specimens with vacuum packing and cooling (VPAC) at $4{ }^{\circ} \mathrm{C}$ as experienced and routinely practiced in our hospital. ${ }^{16-18}$ We have shown that fresh tissues kept vacuum sealed at $4{ }^{\circ} \mathrm{C}$ during transfer from the surgical theatre to the pathology laboratory are

\footnotetext{
IInstitute of Pathology, Technische Universität München, Trogerstrasse, Munich, Germany; ${ }^{2}$ Department of Medical Sciences, University of Turin, Via Santena, Turin, Italy; ${ }^{3}$ Pathology Service, Azienda Ospedaliera Città della Salute e della Scienza di Torino, Via Santena, Turin, Italy and ${ }^{4}$ Department of Molecular Biotechnologies and Health Sciences, University of Turin, Via Nizza, Turin, Italy

Correspondence: Professor Dr K-F Becker, PhD, Technische Universität München, Institute of Pathology, Trogerstrasse18, Munich, D-81675, Germany

or Professor G Bussolati, MD, Department of Medical Sciences, University of Turin, Via Santena 7, Turin 10126, Italy.

E-mail: kf.becker@lrz.tum.de or gianni.bussolati@unito.it

${ }^{5}$ These authors contributed equally to this work.
}

Received 9 October 2014; revised 9 December 2014; accepted 31 December 2014 
optimally preserved for the primary cell cultures ${ }^{18}$ and maintain the integrity of nucleic acids (DNA and RNA). ${ }^{19}$ In recent studies, it has also been shown that the fixation of VPAC tissues in cold formalin $\left(4^{\circ} \mathrm{C}\right)$ followed by ethanol at $4{ }^{\circ} \mathrm{C}$ guarantees an acceptable histology, a better preservation of RNA compared with the standard formalin fixation, ${ }^{11}$ possibly by slowing down the enzymatic activity, and an optimal preservation of antigens for IHC analyses. ${ }^{10,20}$

However, the definition of the impact of the pre-analytical variables on protein preservation is rather controversial, in part because of the semi-quantitative nature of IHC scoring. A quantitative extraction-based measurement of protein biomarkers in tissues would most likely be more precise, although the correlation between protein abundance and histology is lost. ${ }^{21}$

In this study, we have taken advantage of a well-defined model of breast cancer, the BALB-neuT mouse. All BALBneuT females are genetically pre-destined to develop, at 100\% penetrance, multiple, uniform, and small mammary tumors expressing HER2 and pAKT proteins at high levels. ${ }^{22}$ Notably, mammary carcinogenesis in BALB-neuT mice displays consistent, stepwise and directly age-related progression that mimics several features of the human breast cancer. ${ }^{23,24}$ The multiple cancers over-expressing rat neu protein ${ }^{25}$ and the phosphorylated form of $\mathrm{AKT}^{22}$ arise at $\sim 3$ months of age, and their subcutaneous location allows a fast collection and processing under different experimental conditions.

Using this model and thus a series of biologically identical tumors, we have performed a series of experiments to evaluate the combined and relative effect of time and temperature before and during fixation on the preservation of biomarkers in breast cancer. Protein biomarkers of interest (eg, HER2 and several phosphoproteins) were examined with an extraction-based quantitative evaluation methodology, namely reverse phase protein arrays (RPPA). In parallel, and to assess the clinical and diagnostic value of the data accrued in the experimental model, studies were conducted in a series of human breast cancers, both in core biopsies and related surgical specimens fixed in formalin either using standard conditions or following a cold formalin protocol.

\section{MATERIALS AND METHODS}

\section{Standard Fixation (SF) Procedure}

Samples (4 mm thick) were fixed for $24 \mathrm{~h}$ in $4 \%$ neutralbuffered formalin (NBF) (Histo-Line Laboratories, Milan, Italy) at room temperature (RT), routinely processed for paraffin embedding with an automatic processor (Leica ASP 300, Leica Microsystems, Wetzlar, Germany) and embedded in paraffin wax (FFPE).

\section{Cold Fixation (CF) Procedure}

Samples were immersed in precooled 4\% NBF (Histo-Line Laboratories, Milan, Italy) at $4{ }^{\circ} \mathrm{C}$ for $24 \mathrm{~h}$ (using the ColdFIX ${ }^{\circledR}$ apparatus by Milestone Srl, Bergamo, Italy). Thereafter, the specimens were transferred to ethanol $95 \%$ at $4{ }^{\circ} \mathrm{C}$ for $4 \mathrm{~h}$ to keep enzyme activity inhibited during the time of ethanol penetration. ${ }^{11}$ The tissues were processed for paraffin embedding by using the same processor (Leica ASP 300, Leica Microsystems, Wetzlar, Germany) and following the same program used for the standard FFPE, but skipping the first $95 \%$ ethanol step.

\section{Mouse Samples}

BALB-neuT male mice ${ }^{25}$ from Biogem (Ariano Irpino, Italy) were crossed with $\mathrm{BALB} / \mathrm{c}$ female mice purchased from Charles River Laboratories (Calco, Italy). The progeny was genotyped to discriminate between wild type $\mathrm{BALB} / \mathrm{c}$ and BALB-neuT females heterozygous for the neu transgene. All mice were bred under specific pathogen-free conditions (Allentown Caging Equipment, Allentown, NJ, USA) at the Molecular Biotechnology Center (Torino, Italy) and treated according to the European Guidelines and policies, as approved by the University of Torino Ethical Committee. To assess mammary tumor growth, BALB-neuT females were inspected weekly by palpation, and progressively growing masses with a mean diameter of $>1 \mathrm{~mm}$ were considered tumors. Growth was monitored until all ten mammary glands displayed a tumor or until a tumor exceeded a mean diameter of $10 \mathrm{~mm}$, time at which the mice were sacrificed.

Immediately after excision, six tumor samples were cut at $4-\mathrm{mm}$ thick and directly fixed in formalin at RT (standard formaline $(\mathrm{SF})$ ) or at $4{ }^{\circ} \mathrm{C}(\mathrm{CF})$ and paraffinembedded. To evaluate the effect of cold ischemia, 57 samples were stored at RT or at $4{ }^{\circ} \mathrm{C}$ for different times up to $24 \mathrm{~h}$ and then cut at $4 \mathrm{~mm}$ and either fixed following SF or $\mathrm{CF}$ before paraffin embedding (Figures 1a and 2a and b). Each of the different experimental steps was performed in triplicate.

\section{Human Samples}

The study was approved by the 'Comitato per l'utilizzo dei campioni biologici di derivazione umana-Comittee for human Biospecimen Utilization (DSM-ChBU), of the Department of Medical Sciences, University of Turin, Italy. Written informed consent was obtained from all patients for their tissue to be used in research.

Human breast cancer specimens were collected from the Pathology Service of the Azienda Ospedaliera Universitaria Città della Salute e della Scienza di Torino, Italy. Pre-surgical core biopsies of invasive breast cancer of no special type (ICNST) obtained from six different patients were immediately fixed in SF before paraffin embedding. Following the VPAC procedure (Tissue-Safe ${ }^{\circledR}$, Milestone Srl) allowed for routine use in our hospital for surgical specimens transfer, ${ }^{17}$ the matched resection specimens of breast cancers were stored under vacuum in a fridge at $4{ }^{\circ} \mathrm{C}$ till grossing. Two samples of each surgical specimen were then fixed in parallel in SF (total of six samples) and in CF (total of six samples) and then paraffin embedded (Figures $1 \mathrm{~b}$ and $2 \mathrm{c}$ and $\mathrm{d}$ ). 
a

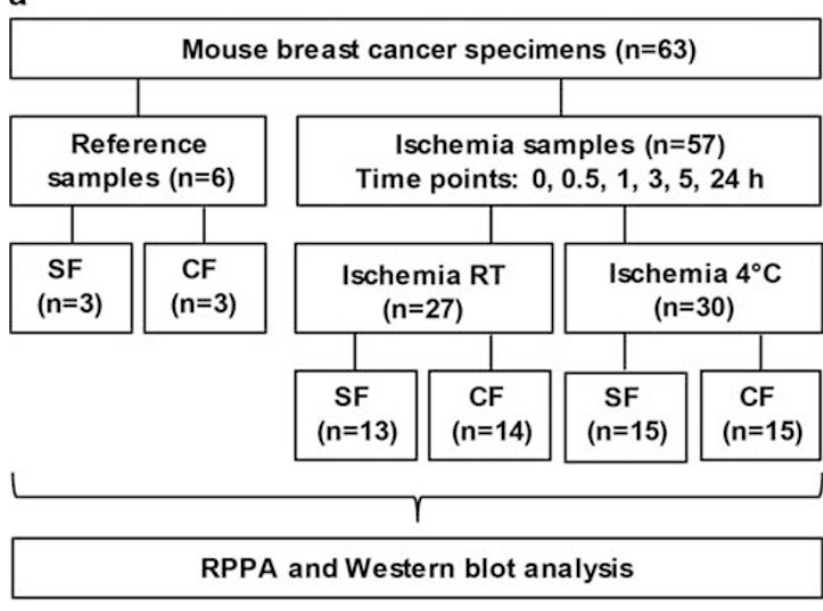

b

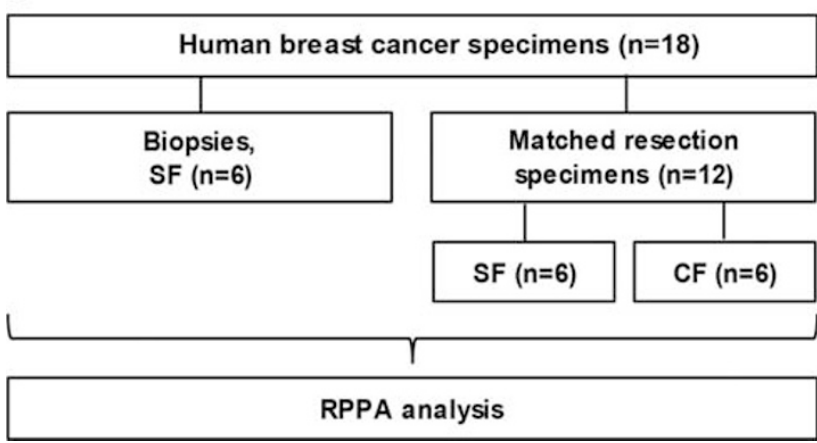

Figure 1 Study design. (a) Murine breast cancer specimens were collected. The reference samples were immediately fixed in standard formalin (room temperature) (SF) or cold formalin $\left(4^{\circ} \mathrm{C}\right)$ (CF) and paraffin embedded. The ischemia samples were stored at room temperature or under vacuum at $4{ }^{\circ} \mathrm{C}$ during the ischemia time up to $24 \mathrm{~h}$ and were fixed in either standard formalin or cold formalin before paraffin embedding. (b) Human breast cancer specimens were collected. Biopsies were immediately fixed in standard formalin before paraffin embedding. The matched resection specimens were stored under vacuum at $4{ }^{\circ} \mathrm{C}$ for $20 \mathrm{~h}$ before preservation and fixed either in standard formalin or cold formalin before paraffin embedding. Proteins were analyzed by reverse phase protein array and/or western blot analysis.

In addition to the histological examination for diagnosis, all cases underwent IHC evaluation for estrogen (ER) (rabbit $\mathrm{mAb}$, clone SP1, Ventana-Diapath, Tucson, AZ) and progesterone receptors (PgR) (rabbit $\mathrm{mAb}$, clone 1E2, Ventana-Diapath) and HER2 expression. HER2 status was evaluated by using the Herceptest (Kit Dako). Equivocal (score 2+) cases were investigated by FISH assay (Vysis, Downers Grove, IL, USA). Assessment of IHC results of hormone receptor and HER2 was performed according to the ASCO/CAP guidelines. ${ }^{26,27}$

The hematoxylin \& eosin (H\&E) staining of each tissue specimen (mouse and human) was evaluated by experienced pathologists from the University of Turin (GB) and the Institute of Pathology of the Technische Universität München (ED). Tumor areas were marked for manual dissection and areas with necrosis were excluded. The tumor cell content was at least $80 \%$ in the resection specimens and $20-80 \%$ in the core needle biopsies.

\section{Protein Extraction}

Proteins from FFPE tissues were extracted using the Qproteome FFPE Tissue Kit (Qiagen GmbH, Hilden, Germany) as previously described. ${ }^{28}$ Briefly, $10-\mu \mathrm{m}$ FFPE tissue sections were deparaffinized, and proteins were extracted by using EXB Plus buffer (Qiagen GmbH, Hilden, Germany), which was supplemented with protease (Complete Protease Inhibitor Cocktail), phosphatase (PhosSTOP Phosphatase Inhibitor Cocktail, Roche Applied Science, Mannheim, Germany) and kinase inhibitors (staurosporine and genistein, Sigma-Aldrich Chemie GmbH, Munich, Germany). Protein concentration was determined by using the Bradford protein assay (BioRad, Hercules, USA) according to the manufacturer's instructions. For generating protein arrays, the concentration was adjusted to $2 \mathrm{mg} / \mathrm{ml}$. Protein lysates were stored at $-20^{\circ} \mathrm{C}$ short-term and for long-term storage at $-80^{\circ} \mathrm{C}$.

\section{Analysis of Protein Expression by Reverse Phase Protein Arrays (RPPA)}

RPPAs were generated by using the SpotBot Extreme microarrayer according to the manufacturer's instructions (Arrayit, Sunnyvale, CA 94089, USA). Briefly, for every lysate and every dilution (not diluted, 1:2, 1:4, 1:8, 1:16, buffer), two replicates were applied onto a nitrocellulose-coated glass slide (Oncocyte Avid Grace Bio-Labs, Bend, USA), which produced 12 data points per sample. These miniature dilution curves allow analysis of each analyte/antibody combination in the linear dynamic range. ${ }^{29}$ The slides were incubated with specific antibodies (Supplementary Table 1), and proteins were visualized by using a mixture of ECL Prime Western Blotting Detection Reagents and ECL Advance Western Blotting Detection Kit (Amersham/GE Healthcare Europe GmbH, Freiburg, Germany). The developed films were scanned individually on a scanner (Scanjet 3770, Hewlett-Packard, Hamburg, Germany) with 1200 dpi and saved as TIF files. The TIF images were analyzed by using MicroVigene 3.5.0.0 (VigeneTech, Carlisle, USA) to determine the background corrected intensities for each spot. For normalization of the antibody signals, the total protein amount was determined by staining representative slides with SYPRO Ruby Protein Blot Stain (Molecular Probes, Eugene, OR, USA); the representative slides were printed at the beginning or at the end of the arraying process. SYPRO Ruby staining was performed according to the manufacturer's instructions and visualized using an E-BOX VX2 UV Transilluminator (Peqlab, Erlangen, Germany). All antibodies used in this study were validated for specificity by western blot analysis.

In analogy to a previous study, ${ }^{29}$ in this study, the interarray variation between two independently spotted arrays was determined. The average inter-array coefficient of variation (CV) ranged between 10 and 12\% (unpublished data and Supplementary Figure 1). 



Figure 2 Breast cancer specimens fixed alternatively in cold formalin (a, c) or in neutral-buffered formalin at room temperature and stained with hematoxylin \& eosin. Figures a and b: breast cancer from BALB-neuT mice. (a) Time 0 specimens, fixed in cold formalin. (b) Specimen with cold ischemia time of $5 \mathrm{~h}$ at room temperature, followed by standard neutralbuffered formalin fixation at RT. Please notice that morphology is similar while, as shown in Figure 3, the preservation of phosphoprotein markers is dramatically different. (c and d) Human breast carcinoma (same case), fixed alternatively in cold formalin (c) or in formalin at room temperature (d). The histological features are not affected by the temperature of the fixative (magnification: $200 \times$; insets in figures a and b: $400 \times$ ).

\section{Western Blot (WB) Analysis}

Equal amounts of protein lysates $(25 \mu \mathrm{g})$ were separated by one-dimensional SDS-PAGE and blotted onto nitrocellulose membranes. Protein detection was performed using antip-Erk-1/2, anti-p-Akt, anti-p-HER2, and anti-beta-actin antibodies (Supplementary Table 1). Secondary antibodies used for RPPA and western blot analysis were anti-rabbit IgG, HRP-linked antibody from Cell Signaling Technology (\#7074) and anti-mouse IgG, HRP-linked antibody from GE Healthcare (NA931). Proteins were visualized by using ECL solution. Western blot quantification was performed by using the open source software Image J (National Institutes of Health, USA).

\section{Statistics}

Statistical analysis of the normalized data received by RPPA was performed by using the parametric $t$-test. All statistical analyses were conducted two-sided, and a $P$-value $<0.05$ was considered to indicate statistical significance by using SPSS version 22.0 (SPSS, Chicago, IL, USA).

\section{RESULTS \\ Protein and Phosphoprotein Preservation of Mouse Samples}

A total of 63 mammary tumors from 31 BALB-neuT mice were fixed in formalin and processed for paraffin embedding (Figure 1). Histological examination showed a uniform pattern of invasive carcinomas growing as solid nests and clumps of cancer cells with a monotonous cytology. Scattered foci of necrosis were only occasionally detected.

We analyzed 25 proteins of biological and clinical interest for breast cancer, focusing our interest on the PI3 kinase/AKT pathway (Table 1).

First, we investigated the levels of nine proteins: HER2, ER, PgR, AKT, Cyclin D, mTOR, and Cytokeratin 18, and two reference proteins, GAPDH and beta-Actin. In all cases, the antibodies used (and employed for the analysis of human samples as well; see below) showed an extensive cross-reactivity with the mouse proteins. The HER2 protein over-expressed in these experimental tumors was analyzed by using two reagents commonly used for IHC evaluation in human breast cancers: the DAKO polyclonal serum A0485 and the CB11 monoclonal antibody Novocastra. Both reagents were reactive, and we were thus able to establish the effect of differential experimental conditions of cold ischemia time, temperature and fixation on the preservation of the protein. The RPPA data indicate that the HER2 protein, at least as detectable through the epitopes analyzed, is relatively robust and insensitive to delayed tissue processing, by using room or cold temperature preservation. Signals were preserved even after several hours (up to 5) of ischemia time, while a partial decrease was observed only when the delay to fixation was extended to $24 \mathrm{~h}$ in cold conditions (Supplementary Table 2). The polyclonal reagent gave a stronger signal than the CB11 monoclonal antibody, and the difference was more marked in samples processed after prolonged ischemia time. Among the other proteins, PgR and mTOR produced variable results, whereas the ER values were stable across the different preservation conditions, which was similar to those observed for HER2.

Fixation in CF, as opposed to SF, allowed a satisfactory preservation of proteins. The difference was not statistically significant, but a trend was observed pointing to a better preservation fixing at $4{ }^{\circ} \mathrm{C}$.

Next, we focused on 16 phosphoproteins, selected for their biological and clinical relevance to breast cancer and because they represented major signaling pathways (eg, PI3K and MAPK pathways). Although sensitivity to ischemia was a constant feature for almost all of the analyzed phosphorylated proteins, its degree varied (Table 1, Figure 3a). Some phosphoproteins of diagnostic interest, such as pHER2 and pAKT, showed an extreme sensitivity, with a $>75 \%$ loss of the respective signal intensity as the result of ischemic conditions. 
The effect was already critical after $30 \mathrm{~min}$ of ischemia, a finding fitting with the challenge of human resection samples for the detection of these markers (see below). Other phosphoproteins (eg, pHER3, pS6RP, pSTAT3, and pSTAT5) were instead found to be rather insensitive to ischemic conditions (Table 1).

CF allowed a statistically significant improvement in the preservation of ischemia-sensitive phosphoproteins, as demonstrated by a loss of signal of $>35 \%$ for pHER2, p-p38 MAPK and pNFkB in specimens fixed in SF compared with parallel tissues fixed in CF (see Supplementary Table 2).

This effect was quite definite at time point zero, that is, immediately fixed after sacrifice of the animals, and was still detectable in specimens that had been kept at $4^{\circ} \mathrm{C}$ for different times (up to $5 \mathrm{~h}$ ). On the contrary, the 'cold fixation advantage' was lost, at any time, in specimens preserved at RT. In addition, an ischemia time at RT, even as short as $30 \mathrm{~min}$, implied a loss of sensitive phosphoproteins, which could not be restored by cold formalin processing (Table 2, Figure $3 \mathrm{~b}$ and $\mathrm{c})$.

\section{Protein and Phosphoprotein Preservation of Human Samples}

Out of six breast cancers, four cases were found to overexpress HER2 protein (score $3+$ ), while two cases (cases \#3 and \#5) were scored $2+$ and HER2 was not amplified as determined by FISH analysis. ER was positive (values ranging from 70 up to $99 \%$ positivity) in all but one case (case \#1), while PgR was positive in four cases and negative in two (cases \#1 and \#6). The staining was performed on sections from blocks of core biopsies and those of surgical specimens subjected to SF and CF fixation processing, and a perfect matching of the results was observed.

Proteins extracted from sections of each case (three different blocks) underwent the same analytical procedures (RPPA and WB) for the same 9 proteins and 16 phosphoproteins as above (Table 1, Supplementary Table 3 ). Because we were interested in the comparative values (and the related effect of the different processing procedures), we set as $100 \%$ the value observed for $\mathrm{CB}$, while the value obtained with the SF and CF material from the same case was expressed as a percentage of the former.

No statistically significant difference, for any marker, was observed when the results for SF vs CF tissue blocks from the resection specimens were analyzed.

Sensitivity to ischemic conditions was therefore determined by comparing data of $\mathrm{CB}$ to those obtained in the resection specimens from the same case. Among the proteins analyzed, only HER2 was found to be sensitive, while the other proteins (and specifically ER and PgR) were found to be insensitive to ischemic conditions following resection, at least in our VPAC conditions. The sensitivity of HER2 was detected as low $(25 \%$ loss of signal in one or more resection) in samples analyzed with the DAKO polyclonal serum and moderate (50\% loss) when the analysis was performed with the CB11 monoclonal antibody.

The data obtained for the 16 phosphoproteins matched those obtained for the murine samples. In Figure 4, the results of 6 examples are shown. Some phosphoproteins (such as pAKT, pEGFR and pPRAS40) were found to be affected by ischemia with a loss of signal in $>75 \%$ of resections, while others were stable and not affected (eg, pSTAT5). It should be noted that not all patients showed over-expression of the phosphoproteins analyzed; eg, high levels of p-Akt and p-EGFR are only seen in patients \#3 and \#4. In these two patients a dramatic loss of both phosphoproteins is observed in the resection specimens compared to the core biopsies.

\section{DISCUSSION}

The present study was performed to determine the impact of ischemic and fixation steps on the preservation of 16 phosphoproteins and 9 total proteins that are relevant for breast cancer. The data presented here draw significance out of a cross-validation of results obtained in a mouse experimental model and in human material (breast cancer biopsies and resection specimens).

The interest in precise measurements of protein biomarkers, including phosphoproteins, is linked to their critical role in predicting the evolution and response to treatment of human tumors, an issue that is highly relevant in breast cancer, where the therapy is currently based on the expression levels of ER, PgR, and HER2, as well as the expression of the proliferation marker Ki67. ${ }^{30-33}$

Recently, studies have highlighted the critical role of the phosphatidylinositol 3-kinase (PI3K)/Akt/mammalian target of rapamycin (mTOR) pathway in mediating key functions that determine the tumor aggressiveness, ${ }^{34}$ and new drugs targeting activated AKT are very promising and are presently being tested. ${ }^{35}$ Therefore, there is much interest in a reliable determination of pAKT in cancer specimens because this is going to be pivotal in the planning of clinical trials and the selection of patients for treatment.

Notoriously, the preservation of tissue components is heavily dependent on the time and conditions (eg, temperature) preceding fixation (so called cold ischemia), ${ }^{13,36}$ and several studies have already established that the best preservation conditions are guaranteed by immediate fixation of histological specimens in formalin, as commonly practiced in pre-surgical diagnostic procedures such as core biopsies for breast cancer. ${ }^{37,38}$ However, we and others previously reported that the temperature of the fixative is also an important parameter to be considered. ${ }^{10,11}$

In the present experimental study, we took advantage of the genetic predestination of female BALB-neuT mice to develop HER2 protein ${ }^{39}$ and pAKT $^{22}$ over-expressing spontaneous invasive carcinomas at determined time points in multiple mammary glands. By exploiting this experimental material, we were able to assess the impact of different technical variables (time, temperature, and fixation procedures) on the 
Table 1 Summary of protein and phosphoprotein sensitivities to ischemia in the murine and human data sets

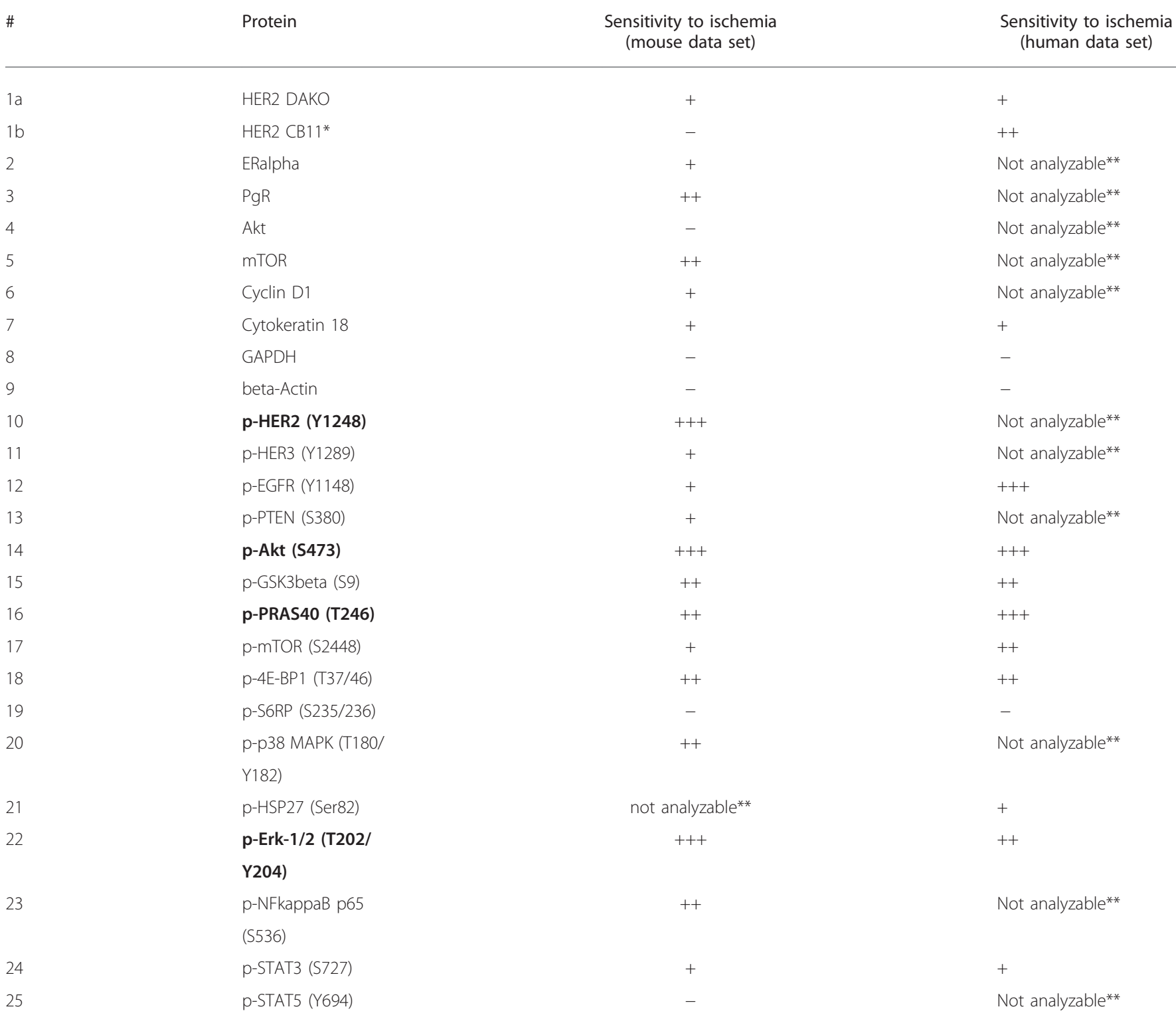

Mouse data set.

The results are depicted as the mean normalized signal intensities, relative to the reference sample $(0 \mathrm{~h}$ ischemia) which was set as $100 \%$; detailed data are shown in Supplementary Table 2; the $24 \mathrm{~h}$ time point was excluded because after $24 \mathrm{~h}$ nearly each protein showed massive loss of signal and the other time points are clinically more relevant.

,$+ \geq 25 \%$ Loss of signal in one or more ischemia samples compared with reference sample.

,$++ \geq 50 \%$ Loss of signal in one or more ischemia samples compared with reference sample.

,$+++ \geq 75 \%$ Loss of signal in one or more ischemia samples compared to reference sample.

-, Not sensitive.

*, The antibody clone CB11 against HER2 is a monoclonal antibody; the signals were in general weaker than those revealed by the DAKO polyclonal antibody.

** Protein level was too low, no quantification possible.

Bold, most ischemia sensitive proteins.

Human data set.

The results are depicted as the normalized signal intensities, relative to the reference sample (biopsy) which was set as $100 \%$ (detailed data are shown in Supplementary Table 3).

,$+ \geq 25 \%$ loss of signal in one or more resection samples $v s$ respective biopsy.

,$++ \geq 50 \%$ loss of signal in one or more resection samples vs respective biopsy.

,$+++ \geq 75 \%$ loss of signal in one or more resection samples $v s$ respective biopsy.

-, not sensitive.

** Protein expression was too low, no quantification possible. 

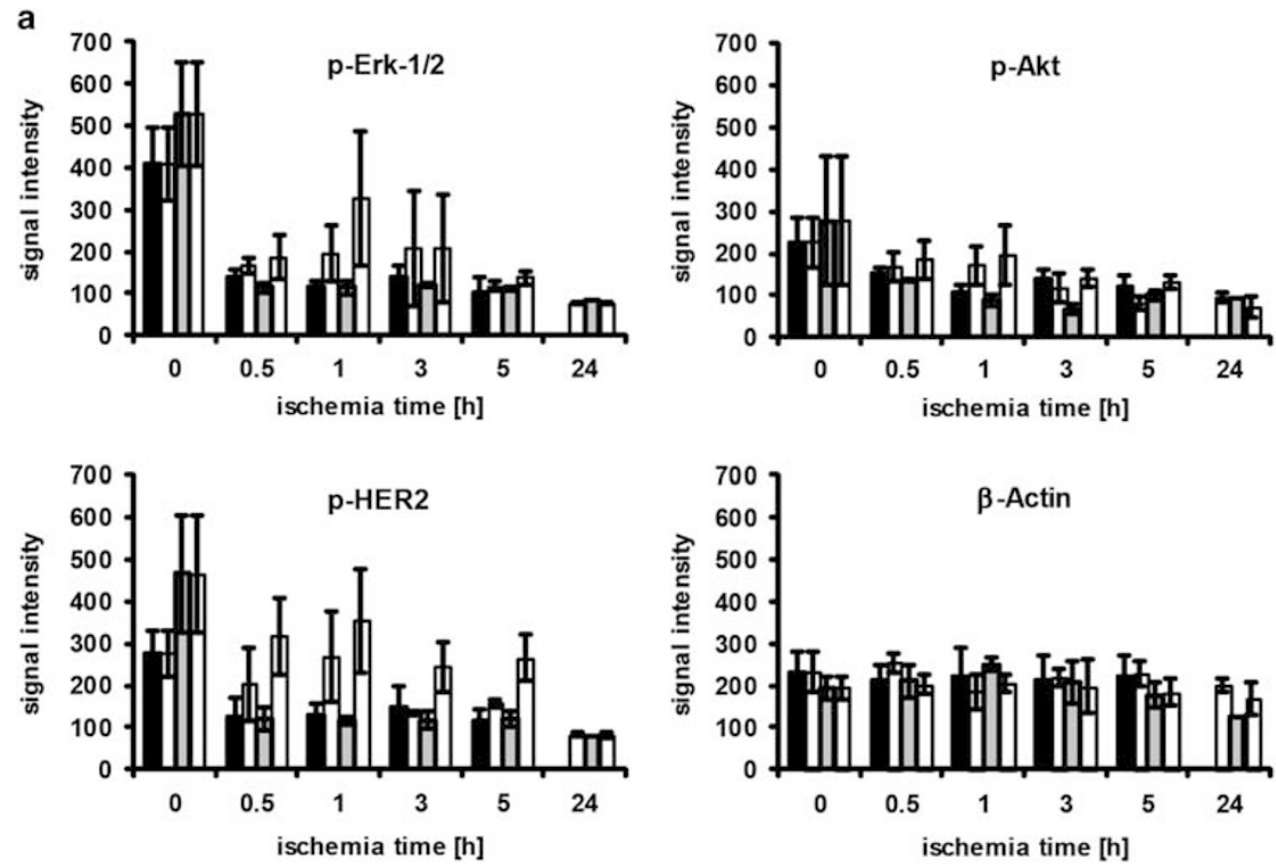

$\mathrm{SF}$, ischemia RT $\square \mathrm{CF}$, ischemia RT

$\square \mathrm{SF}$, ischemia $4^{\circ} \mathrm{C} \quad \square \mathrm{CF}$, ischemia $4^{\circ} \mathrm{C}$

Figure 3 Loss of phosphoprotein signal due to delayed preservation (murine samples). Murine breast cancer specimens were either fixed in standard formalin or cold formalin immediately after resection or after different ischemia time points with storage until preservation at room temperature or under vacuum at $4^{\circ} \mathrm{C}$. Protein levels were either determined by reverse phase protein arrays (a and $\left.\mathbf{b}\right)$ or by western blot analysis (c) with the depicted antibodies. The reverse phase protein arrays data in (a) are shown as normalized signal intensities that were calculated by normalization to total protein (SYPRO Ruby staining). Each bar in the reverse phase protein arrays study reflects $n=3$ data points, besides setting 'cold formalin, ischemia room temperature', time point $5 \mathrm{~h}$ reflects $n=4$ and time point $24 \mathrm{~h}$ reflects $n=1$. For the setting 'standard formalin, the ischemia room temperature' time point $24 \mathrm{~h}$ is missing because it was not analyzable. In (b), two examples (pAKT and pHER2) are highlighted showing the advantage of cold formalin if specimens are kept at $4^{\circ} \mathrm{C}$ during the cold ischemia duration. Data are shown as signal intensities in percent relative to the time point zero. Western blot data were normalized to the reference protein beta-Actin before quantification.

preservation of protein biomarkers, including HER2 and pAKT. A standardized study of these issues cannot consistently be planned and conducted in human material, whereas as shown here, the approach of studying tumors from BALB-neuT mice was feasible.

Moreover, the RPPA is a reliable quantitative technology to assess the impact of different technical variables (time, temperature, and fixation procedures) on the preservation of biological markers and has the potential to be incorporated into the routine clinical workflow..$^{40}$ Our approach allowed us to transfer the results of the animal model to humans to directly address questions of diagnostic and clinical relevance. However, whereas the mouse cancers showed a uniform structure consisting of nests of cancer cells making up more than $80 \%$ of the tumors and provided fully reproducible data, the results from the human material were less consistent, most likely because of inter-patient variability and discrepancies in tumor type and composition.

In the experimental model employed here, BALB-neuT mice are transgenic for an activated form of the rat neu gene (showing an $88 \%$ homology with the human HER2 gene) expressed under the control of the mouse mammary tumor virus promoter long terminal repeats (MMTV-LTR promoter). The single-point mutation at position 664 in the transmembrane domain of the rat neu receptor results in the formation of homo- and heterodimers that spontaneously transduce the proliferative signals that are responsible for the transformation of mammary epithelial cells. ${ }^{25}$ In this experimental murine model, the over-expressed HER2 protein was fully reactive with both immunological reagents currently employed to study HER2 expression in human breast cancer, ie, the rabbit polyclonal serum (from DAKO) and the CB11 monoclonal antibody. This allowed us to experimentally investigate the effect of ischemia time and temperature on the preservation of this protein.

Consistent with previous observations by our group, ${ }^{12}$ the preservation of HER2 protein, as quantitatively evaluated with the RPPA procedure, was not significantly affected by the fixation procedure (standard or cold formalin) or the length of cold ischemia time up to $5 \mathrm{~h}$, independent of the temperature and the targeted antigen. However, the polyclonal DAKO antiserum provided a stronger signal than the CB11 monoclonal antibody, and the difference was more pronounced in samples processed after a prolonged ischemia time. This seems 



Figure 3 (Continued)

\section{Table 2 Quantification of western blot results}

\begin{tabular}{lccc}
\hline $\begin{array}{c}\text { Relative signal } \\
\text { intensity } \\
\text { difference } \\
\text { between SF vs CF } \\
\text { reference (CF set } \\
\text { as 100\%) }\end{array}$ & $\begin{array}{c}\text { Setting: SF, ischemia } \\
4^{\circ} \mathrm{C} \text { Relative signal } \\
\text { intensity difference } \\
\text { between reference } \\
\text { (set as 100\%) and } \\
\text { ischemia samples }\end{array}$ & $\begin{array}{c}\text { Setting: CF, ischemia } \\
4{ }^{\circ} \mathrm{C} \text { Relative signal } \\
\text { intensity difference } \\
\text { between reference } \\
\text { (set as 100\%) and } \\
\text { ischemia samples }\end{array}$ \\
\hline p-Erk-1/2 & 38 & 4 & 24 \\
p-Akt & 24 & 5 & 27 \\
p-HER2 & 34 & 6 & 39
\end{tabular}

All signal intensities of the antibodies were normalized to the reference protein $\beta$-Actin. The results of the ischemia samples are depicted as the mean normalized signal intensity over all ischemia samples.

to fit with a heterogeneous sensitivity to ischemic conditions of the different HER2 epitopes and suggests that when dealing with breast cancers with equivocal or undefined preservation conditions, the use of the polyclonal reagent might represent a safer diagnostic alternative.
In the human material, similar and parallel results were obtained when studying core biopsies and related surgical specimens, either standard or cold fixed. Again, for HER2, the duration of the cold ischemia time was not found to be a critical factor, but the breast cancer specimens have routinely been processed by using the VPAC procedure, ${ }^{16,17}$ which is known to achieve very good preservation of biomarkers up to several hours after surgery in breast cancer specimens. ${ }^{20}$

An important result of our study was the finding that some phosphoproteins are more sensitive to prolonged cold ischemia time than others. For example, pHER2 and pAKT were very sensitive, whereas pS6RP and pSTAT5 were relatively stable. This result is consistent with data from the literature; Baker et al. ${ }^{14}$ and Bonnas et al..$^{41}$ found a marked reduction in PAKT in xenograft models when the cold ischemia time was longer than $30 \mathrm{~min}$. Based on comparisons of the phosphoprotein levels in core needle biopsies and resection specimens, we found a marked decrease in many phosphoproteins in the resection specimens, most likely because of the almost complete absence of a cold ischemia time in biopsies compared with the complex processing of the 
GF, biopsy $\square \mathrm{CF}$, resection specimen $\square \mathrm{SF}$, resection specimen
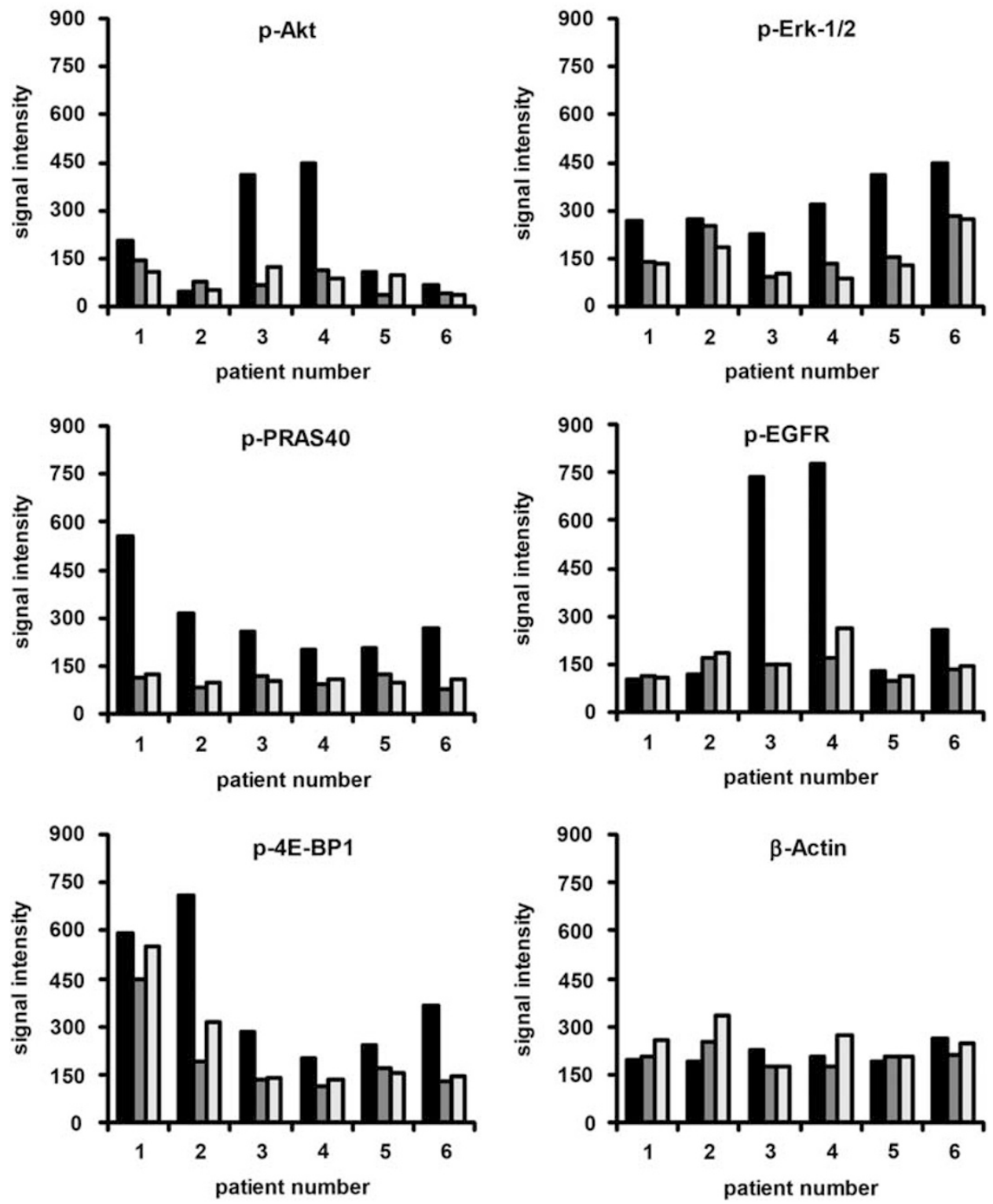

Figure 4 Loss of phosphoprotein signal due to delayed preservation (human samples). Human breast cancer biopsies from six patients were immediately fixed in standard formalin, and matched resection specimens were either fixed in standard formalin or cold formalin. Protein expression was determined by reverse phase protein arrays with depicted antibodies. The reverse phase protein arrays data are depicted as normalized signal intensities, which were calculated by normalization to total protein (SYPRO Ruby staining).

surgical specimens. Surgical specimens would thus prove to be unsuitable for an accurate measurement of many phosphoproteins. This result, which indicates that small, pre-surgical biopsies, as opposed to resection samples should represent the material of choice for the detection of phosphoproteins of diagnostic and clinical interest, such as pHER2 and pAKT, is consistent with the study by Bai et al. ${ }^{37}$ who used an alternative quantitative assessment procedure. A recent study by MericBernstam et al. ${ }^{42}$ stressed the superior preservation of phosphoprotein markers in core biopsies of breast cancer, compared with the related resection specimens. Even the improved preservation conditions of surgical specimens, as currently practiced in our service through VPAC, cannot prevent the loss of phosphoprotein epitopes because we showed that the cooling down of large surgical specimens is a relatively lengthy process, ${ }^{18}$ which cannot match the critical ischemia time of $<30 \mathrm{~min}$, as established here.

Although on one hand core biopsies seem to be more suitable than surgical specimens to assess phosphoantigens, on the other hand a word of caution should be voiced about the intratumoral heterogeneity of biomarker expression in breast cancer (such as HER2) ${ }^{43,44}$ that may not be completely 
figured out by core biopsy samples. This is of particular importance especially when large lesions have to be evaluated, unless multiple sampling of the lesion is performed. ${ }^{45}$

The overall conclusion from our mouse and human data, although obtained on a limited series of samples, ultimately indicate a critical role of cold ischemia, the temperature before and during tissue fixation, and the specimen type (biopsy vs resection specimen) for preserving sensitive phosphoproteins. Fixation in cold formalin, compared to standard fixation at RT, resulted in a statistically significant improvement in the preservation of the reactivity of pHER2 and pAKT (Figure 3a). A limit of our study is the lack of comparison between RPPA data and IHC data, however, our quantitative evaluation evidence is consistent with the IHC study by Chafin et al. ${ }^{10}$ who recently showed that fixation in cold formalin followed by a further processing with hot fixative allows a better preservation of phosphorylated epitopes compared to the standard procedure.

A rational interpretation of the observed data and conclusions have to take into consideration the following points: (i) formalin penetrates into immersed specimens at a speed of approximately $3.6 \mathrm{~mm}$ in the first hour ${ }^{46}$ and (ii) when tissues are immersed in a relatively large volume of formalin, they achieve the temperature of the solution. These considerations suggest that in relatively large specimens (as here exemplified by the experimental rodent tumors), immersion into cold formalin would result in cooling down of the tissue temperature (and presumably an inhibition of phosphatase activity) while the fixative is penetrating into the tissue. Implementation of this procedure in human specimens would imply fixation in cold formalin just a few minutes after surgical removal, a procedure that does not appear to be easily practical in routine practice.

In small specimens of the size of core biopsies $(1 \mathrm{~mm}$ in diameter), the penetration of the formalin within the threshold time of $15 \mathrm{~min}$ seems to provide a satisfactory preservation of sensitive phosphorylated residues. Further improvements, such as cold fixation of core biopsies or the use of alternative fixatives, may be tested in future experiments.

Supplementary Information accompanies the paper on the Laboratory Investigation website (http://www.laboratoryinvestigation.org)

\section{ACKNOWLEDGMENTS}

We thank the personnel of the surgical theaters of the Breast Unit at Azienda Ospedaliera Città della Salute e della Scienza di Torino for the productive collaboration regarding specimen handling. We thank Stefanie Avril for her help with some of the samples. This work was supported by Ricerca Sanitaria Finalizzata (RF-2010-2310674 to AS), by the Italian Association for Cancer research-AIRC (MFAG13310 to CM) and by the German Federal Ministry of Education and Research (BMBF) Spitzencluster Muenchen m4 Biobank Alliance (GRANT no. 01EX1020D).

\section{DISCLOSURE/CONFLICT OF INTEREST}

The authors declare no conflict of interest.
1. Hewitt SM, Badve SS, True LD. Impact of preanalytic factors on the design and application of integral biomarkers for directing patient therapy. Clin Cancer Res 2012;18:1524-1530.

2. Dowsett M. Preoperative models to evaluate endocrine strategies for breast cancer. Clin Cancer Res 2003;9(1 Pt 2):502S-510S.

3. Pinhel IF, Macneill FA, Hills MJ et al. Extreme loss of immunoreactive $\mathrm{p}$-Akt and $\mathrm{p}$-Erk1/2 during routine fixation of primary breast cancer. Breast Cancer Res 2010;12:R76.

4. Betsou $F$, Barnes R, Burke $T$ et al. Human biospecimen research: experimental protocol and quality control tools. Cancer Epidemiol Biomarkers Prev 2009;18:1017-1025.

5. De Cecco L, Musella V, Veneroni $S$ et al. Impact of biospecimens handling on biomarker research in breast cancer. BMC Cancer 2009; 9:409.

6. Hammond ME, Hayes DF, Dowsett M et al. American Society of Clinical Oncology/College of American Pathologists guideline recommendations for immunohistochemical testing of estrogen and progesterone receptors in breast cancer (unabridged version). Arch Pathol Lab Med 2010;134:e48-e72.

7. Robb JA, Gulley ML, Fitzgibbons PL et al. A call to standardize preanalytic data elements for biospecimens. Arch Pathol Lab Med 2013;138: 526-537.

8. Juhl H. Preanalytical aspects: a neglected issue. Scand J Clin Lab Invest Suppl 2010;242:63-65.

9. Spruessel A, Steimann G, Jung $M$ et al. Tissue ischemia time affects gene and protein expression patterns within minutes following surgical tumor excision. Biotechniques 2004;36:1030-1037.

10. Chafin D, Theiss A, Roberts $E$ et al. Rapid two-temperature formalin fixation. PLoS One 2013;8:e54138.

11. Bussolati G, Annaratone L, Medico E et al. Formalin fixation at low temperature better preserves nucleic acid integrity. PLoS One 2011; 6:e21043.

12. Gundisch S, Hauck S, Sarioglu $\mathrm{H}$ et al. Variability of protein and phosphoprotein levels in clinical tissue specimens during the preanalytical phase. J Proteome Res 2012;11:5748-5762.

13. Gundisch S, Grundner-Culemann K, Wolff $\mathrm{C}$ et al. Delayed times to tissue fixation result in unpredictable global phosphoproteome changes. J Proteome Res 2013;12:4424-4434.

14. Baker AF, Dragovich T, Ihle NT et al. Stability of phosphoprotein as a biological marker of tumor signaling. Clin Cancer Res 2005;11: 4338-4340.

15. Mueller C, Edmiston KH, Carpenter $\mathrm{C}$ et al. One-step preservation of phosphoproteins and tissue morphology at room temperature for diagnostic and research specimens. PLoS One 2011;6:e23780.

16. Bussolati G, Chiusa L, Cimino A et al. Tissue transfer to pathology labs: under vacuum is the safe alternative to formalin. Virchows Arch 2008;452:229-231.

17. Di Novi C, Minniti D, Barbaro $S$ et al. Vacuum-based preservation of surgical specimens: an environmentally-safe step towards a formalinfree hospital. Sci Total Environ 2010;408:3092-3095.

18. Annaratone $L$, Marchio $C$, Russo $R$ et al. A collection of primary tissue cultures of tumors from vacuum packed and cooled surgical specimens: a feasibility study. PLoS One 2013;8:e75193.

19. Comanescu $M$, Annaratone $L, D$ 'Armento $G$ et al. Critical steps in tissue processing in histopathology. Recent Pat DNA Gene Seq 2012;6:22-32.

20. Neumeister VM, Parisi F, England AM et al. A tissue quality index: an intrinsic control for measurement of effects of preanalytical variables on FFPE tissue. Lab Invest 2014;94:467-474.

21. Becker KF, Taylor CR. 'Liquid morphology': Immunochemical analysis of proteins extracted from formalin-fixed paraffin-embedded tissues: Combining proteomics with immunohistochemistry. Appl Immunohistochem Mol Morphol 2011;19:1-9.

22. Porzia A, Lanzardo S, Citti A et al. Attenuation of PI3K/Akt-mediated tumorigenic signals through PTEN activation by DNA vaccine-induced anti-ErbB2 antibodies. J Immunol 2010;184:4170-4177.

23. Green JE, Hudson T. The promise of genetically engineered mice for cancer prevention studies. Nat Rev Cancer 2005;5:184-198.

24. Quaglino E, Mastini C, Forni G et al. ErbB2 transgenic mice: a tool for investigation of the immune prevention and treatment of mammary carcinomas. Curr Protoc Immunol 2008; Chapter 20:Unit 20.9.1-20.9-10.

25. Boggio K, Nicoletti G, Di Carlo E et al. Interleukin 12-mediated prevention of spontaneous mammary adenocarcinomas in two lines of Her-2/neu transgenic mice. J Exp Med 1998;188:589-596. 
26. Wolff AC, Hammond ME, Hicks DG et al. Recommendations for human epidermal growth factor receptor 2 testing in breast cancer: American Society of Clinical Oncology/College of American Pathologists clinical practice guideline update. J Clin Oncol 2013;31:3997-4013.

27. Hammond ME, Hayes DF, Dowsett $M$ et al. American Society of Clinical Oncology/College Of American Pathologists guideline recommendations for immunohistochemical testing of estrogen and progesterone receptors in breast cancer. J Clin Oncol 2010;28: 2784-2795.

28. Wolff C, Schott C, Porschewski P et al. Successful protein extraction from over-fixed and long-term stored formalin-fixed tissues. PLoS One 2011;6:e16353.

29. Berg D, Hipp S, Malinowsky $\mathrm{K}$ et al. Molecular profiling of signalling pathways in formalin-fixed and paraffin-embedded cancer tissues. Eur J Cancer 2010;46:47-55.

30. Lips EH, Mulder L, de Ronde JJ et al. Neoadjuvant chemotherapy in ER+ HER2- breast cancer: response prediction based on immunohistochemical and molecular characteristics. Breast Cancer Res Treat 2012;131:827-836.

31. Moon YW, Park S, Sohn JH et al. Clinical significance of progesterone receptor and HER2 status in estrogen receptor-positive, operable breast cancer with adjuvant tamoxifen. J Cancer Res Clin Oncol 2011; 137:1123-1130.

32. Anderson $H$, Hills $M$, Zabaglo $L$ et al. Relationship between estrogen receptor, progesterone receptor, HER-2 and Ki67 expression and efficacy of aromatase inhibitors in advanced breast cancer. Ann Oncol 2011:22:1770-1776.

33. Tanei T, Shimomura A, Shimazu K et al. Prognostic significance of Ki67 index after neoadjuvant chemotherapy in breast cancer. Eur J Surg Oncol 2011;37:155-161.

34. McAuliffe PF, Meric-Bernstam F, Mills GB et al. Deciphering the role of $\mathrm{PI} 3 \mathrm{~K} / \mathrm{Akt} / \mathrm{mTOR}$ pathway in breast cancer biology and pathogenesis. Clin Breast Cancer 2010;10(Suppl 3):S59-S65.

35. Lin J, Sampath D, Nannini MA et al. Targeting activated Akt with GDC-0068, a novel selective Akt inhibitor that is efficacious in multiple tumor models. Clin Cancer Res 2013;19:1760-1772.

36. Neumeister VM, Anagnostou V, Siddiqui S et al. Quantitative assessment of effect of preanalytic cold ischemic time on protein expression in breast cancer tissues. J Natl Cancer Inst 2012;104:1815-1824.

37. Bai $\mathrm{Y}$, Cheng $\mathrm{H}$, Bordeaux J et al. Comparison of HER2 and phospho-HER2 expression between biopsy and resected breast cancer specimens using a quantitative assessment method. PLoS One 2013;8: e79901.
38. Berg D, Langer $\mathrm{R}$, Tran $\mathrm{K}$ et al. Protein microarray-based comparison of HER2, estrogen receptor, and progesterone receptor status in core biopsies and surgical specimens from FFPE breast cancer tissues. Appl Immunohistochem Mol Morphol 2011;19:300-305.

39. Quaglino E, Rolla $S$, lezzi $M$ et al. Concordant morphologic and gene expression data show that a vaccine halts HER-2/neu preneoplastic lesions. J Clin Invest 2004;113:709-717.

40. Akbani R, Becker KF, Carragher $\mathrm{N}$ et al. Realizing the promise of reverse phase protein arrays for clinical, translational, and basic research: a workshop report: The RPPA (Reverse Phase Protein Array) Society. Mol Cell Proteomics 2014;13:1625-1643.

41. Bonnas C, Specht $K$, Spleiss $O$ et al. Effects of cold ischemia and inflammatory tumor microenvironment on detection of PI3K/AKT and MAPK pathway activation patterns in clinical cancer samples. Int Cancer 2012;131:1621-1632.

42. Meric-Bernstam F, Akcakanat A, Chen $\mathrm{H}$ et al. Influence of biospecimen variables on proteomic biomarkers in breast cancer. Clin Cancer Res 2014; 20:3870-3883.

43. Oakman C, Sapino A, Marchio C et al. Chemotherapy with or without trastuzumab. Ann Oncol 2010;21(Suppl 7):vii112-vii119.

44. Sapino A, Goia M, Recupero $D$ et al. Current challenges for HER2 testing in diagnostic pathology: state of the art and controversial issues. Front Oncol 2013;3:129.

45. Marchio C, Sapino A.. The pathologic complete response open question in primary therapy. J Natl Cancer Inst Monogr 2011;2011: 86-90.

46. Buesa RJ.. Histology without formalin? Ann Diagn Pathol 2008;12 387-396.

(1) $(9$ This work is licensed under a Creative Commons Attribution-NonCommercial-NoDerivs

International License. The images or other third party material in this article are included in the article's Creative Commons license, unless indicated otherwise in the credit line; if the material is not included under the Creative Commons license, users will need to obtain permission from the license holder to reproduce the material. To view a copy of this license, visit http://creativecommons.org/licenses/by-ncnd/4.0/ 